\title{
Hacia una caracterización del álgebra temprana a partir del análisis de los currículos contemporáneos de Educación Infantil y Primaria
}

\section{Towards a characterization of early algebra from the analysis of the contemporary curricula of Early Childhood Education and Primary Education}

\author{
Nataly Pincheira Hauck, ${ }^{1}$ Ángel Alsina ${ }^{2}$
}

\begin{abstract}
Resumen: En este artículo se analiza la incorporación del álgebra temprana en los currículos de Educación Infantil y Primaria de Estados Unidos, Australia, Singapur y Chile. A partir del método de análisis de contenido se ha realizado un estudio comparativo y se han establecido unas primeras categorías de conocimiento para caracterizar el álgebra temprana en ambas etapas educativas: 1) Educación Infantil: experimentación con elementos u objetos a partir del reconocimiento de atributos para establecer relaciones, seriaciones de patrones de repetición (identificación, construcción y representación) y descripción de cambios cualitativos y cuantitativos; 2) Educación Primaria: comprensión de distintos tipos de relaciones y de patrones, uso de símbolos algebraicos y modelos matemáticos para representar situaciones matemáticas, comprensión del cambio y uso de variables para determinar una constante o incógnita. Se concluye que es necesario que los programas de formación de maestros incluyan estos conocimientos para articularlos adecuadamente en las dos etapas educativas.
\end{abstract}

Fecha de recepción: 17 de octubre de 2019. Fecha de aceptación: 30 de noviembre de 2020.

1 Universidad de Girona. Facultad de Educación y Psicología. Departamento de Didácticas Específicas. nataly.pincheira@udg.edu. Girona. España. orcid.org/0000-0002-5051-964X

2 Universidad de Girona. Facultad de Educación y Psicología. Departamento de Didácticas Específicas. angel.alsina@udg.edu. Girona. España. orcid.org/0000-0001-8506-1838 
Palabras claves: álgebra temprana, currículo, pensamiento algebraico, Educación Infantil, Educación Primaria.

\begin{abstract}
This article discusses the incorporation of early algebra in the curricula of Early Childhood and Primary Education in the United States, Australia, Singapore and Chile is analyzed. Based on the content analysis method, a comparative study has been made and the first categories of knowledge have been established to characterize early algebra: 1) Early Childhood Education: experimentation with elements or objects based on the recognition of attributes to establish relationships, seriations based on patterns of repetition (identification, construction and representation) and description of qualitative and quantitative changes; 2) Primary Education: understanding different types of relationships and patterns, using algebraic symbols and mathematical models to represent mathematical situations, understanding change and using variables to determine a constant or unknown. It is concluded that it is necessary for teacher training programs to include this knowledge to adequately articulate it in the two educational stages.
\end{abstract}

Keywords: early algebra, curriculum, algebraic thinking, Early Childhood Education, Primary Education.

\title{
1. INTRODUCCIÓN
}

Numerosas investigaciones dedicadas al estudio del álgebra sugieren la necesidad de ser incorporada en los currículos desde los primeros cursos escolares, dado que su inicio según las propuestas clásicas se considera desde la Educación Secundaria. Con el objeto de distinguir el álgebra propiamente dicha de las actividades generadoras de pensamiento algebraico en las primeras edades de escolarización, se ha acuñado el término Early-Algebra, a partir de ahora álgebra temprana, que intenta introducir modos del pensamiento algebraico desde las primeras edades de escolarización (Carraher y Schliemann, 2007; Kaput, 2000, 2008; Molina, 2009). Esta nueva corriente no considera el álgebra como una asignatura, sino como una manera de pensar y actuar en objetos, relaciones, estructuras y situaciones matemáticas para promover una enseñanza 
fundamentada en la comprensión de las matemáticas (Carpenter, Franke y Levi 2003; Carraher, Schliemann y Brizuela, 2000; Kaput, 1995, 1998, 2000).

A pesar de la importancia que está adquiriendo el álgebra temprana, no existe todavía un consenso en la literatura acerca de su caracterización, ni tampoco de los conocimientos que se incluyen bajo el concepto "álgebra temprana". Sin embargo, son varios los currículos de Educación Infantil y Primaria que han empezado a introducir de forma explícita conocimientos para promover el pensamiento algebraico en las primeras edades. Desde esta perspectiva, nuestro propósito es realizar un estudio comparativo de estos currículos que evidencie qué elementos se consideran para el desarrollo del pensamiento algebraico en las primeras etapas escolares y, más específicamente, qué conocimientos se incluyen para promover el aprendizaje del álgebra temprana o bien cómo evoluciona el álgebra temprana a lo largo de estas etapas.

Desde este prisma, el análisis del currículo resulta relevante tanto para la etapa escolar, ya que determina la formación matemática de los escolares (Rico, Díez, Castro y Lupiáñez, 2011), como para el desarrollo profesional del profesor, puesto que el profesorado debe conocer y manejar las orientaciones curriculares para lograr un aprendizaje efectivo en sus estudiantes. En este sentido, en el marco del modelo Mathematical Knowledge for Teaching (MKT), Ball, Thames y Pelps (2008) destacan la importancia del conocimiento del currículo como un subdominio del conocimiento pedagógico del contenido. Este subdominio se refiere al "conocimiento de los objetivos, contenidos, fines, orientaciones curriculares, materiales y recursos disponibles para la enseñanza, que permiten al profesor guiar su práctica y seleccionar las tareas adecuadas para el aprendizaje de sus estudiantes" (Ball, et al., 2008, p. 391).

Asumiendo, pues, que el profesor debe conocer el currículo de matemáticas para mejorar la enseñanza de los contenidos, nuestro estudio considera como punto de discusión la presencia y evolución del álgebra temprana en los currículos de Educación Infantil y Primaria, dado que durante los últimos años se ha generado una transformación curricular progresiva en este bloque de contenido. Este cambio conforma un desafío para la formación del profesorado, pues resulta indispensable contar con maestros capaces de asumir estos cambios y de empoderar el desarrollo del pensamiento algebraico desde las primeras edades. Desde esta perspectiva, la finalidad de este artículo consiste en analizar la incorporación del álgebra temprana en los currículos de Educación Infantil y Primaria y caracterizarla, con el propósito de poder establecer orientaciones específicas acerca de la enseñanza del álgebra temprana y, de esta forma, contribuir al desarrollo 
profesional del profesorado. En concreto, se realiza un estudio comparativo a nivel transversal de las orientaciones curriculares de Educación Infantil y Educación Primaria de Estados Unidos, Australia, Singapur y Chile. Para la selección de estos currículos se ha considerado en primer lugar Estados Unidos, pues es la fuente de referencia del currículo latinoamericano, junto con otros países donde el estudio del álgebra temprana se encuentra de forma explícita en el currículo escolar.

\section{2. ÁLGEBRA TEMPRANA}

El álgebra temprana forma parte de una propuesta de cambio curricular que emerge como resultado de diversos estudios que se han desarrollando durante las últimas décadas (Bastable y Schifter, 2007; Carraher y Schliemann, 2007; Kaput, 1998, 2000; Kaput, Carraher y Blanton, 2009). Esta nueva corriente plantea introducir el desarrollo del pensamiento algebraico principalmente desde la Educación Primaria, aunque algunos organismos y autores proponen su incorporación desde niveles inferiores (Davis, 1985; Vergnaud, 1988; Kaput y Blanton, 2001; Carpenter, Franke y Levi, 2003; NCTM, 2003). El motivo de esta propuesta surge ante la necesidad de eliminar la tardía incorporación del estudio del álgebra en la educación secundaria (Carpenter et al., 2003; Kaput 1998).

Kaput (2000) denomina a este proceso como "la algebrización" del currículo, es decir, la integración del razonamiento o pensamiento algebraico a lo largo de toda la escolaridad. Cabe destacar que el introducir el desarrollo del pensamiento algebraico en los primeros cursos de escolarización no significa impartir un curso específico de álgebra, sino más bien "capacitar a los estudiantes mediante el fomento de un mayor grado de generalidad en su pensamiento y una mayor capacidad de comunicar dicha generalidad" (Lins y Kaput, 2004, p. 58). El obtener buenos resultados dependerá de cómo se piense el currículo escolar, pues no existirán cambios si se aprecia como un listado de contenidos, mientras que existirán cambios profundos si se considera como un conjunto de experiencias para los estudiantes (Kilpatrick, 2011).

En este sentido, el propósito que persigue el álgebra temprana no se fundamenta sólo en preparar a los estudiantes para el estudio del álgebra en niveles educativos superiores, sino desarrollar en ellos modos de pensamiento que les permitan alcanzar una comprensión profunda y compleja de las matemáticas escolares, de manera que éstos permeen en otros bloques de contenido como numeración, medida, geometría, etcétera. 
Según Blanton y Kaput (2005) el álgebra temprana busca promover en las aulas de clase hábitos de pensamiento que atiendan a la estructura que subyace a las matemáticas, por medio de actividades dirigidas a la observación de patrones, relaciones y propiedades matemáticas. Así también, se debe propiciar un ambiente escolar en donde los alumnos exploren, modelicen, hagan predicciones, discutan, argumenten, comprueben ideas e igualmente practiquen habilidades de cálculo.

Del mismo modo, resulta interesante profundizar en la propuesta que plantea Kieran para abordar el desarrollo del pensamiento algebraico:

El pensamiento algebraico en los primeros cursos académicos implica el desarrollo de diversos tipos de reflexiones como parte de las actividades en los que puede utilizarse la representación simbólica algebraica mediante letras como herramienta, pero no exclusiva, del álgebra, de modo que pueda llevarse a cabo también sin ningún tipo de representación simbólica con letras, como por ejemplo el análisis de las relaciones entre cantidades, identificar estructuras, estudiar el cambio, la generalización, la resolución de problemas, el modelado, la justificación, el ensayo y error y la predicción. (Kieran, 2004, p. 149)

Por otra parte, Radford (2011) asegura que "el pensamiento algebraico temprano se basa en las posibilidades del estudiante de comprender patrones en formas co-variantes desarrolladas culturalmente y utilizarlos para tratar cuestiones de términos remotos y no específicos" (p. 23), es decir, para alcanzar un desarrollo del pensamiento algebraico temprano los estudiantes deben identificar regularidades relacionando tanto estructuras numéricas como espaciales.

Estos planteamientos permiten ampliar y considerar nuevos conocimientos y actividades que faciliten el proceso de enseñanza-aprendizaje del álgebra temprana desde las primeras edades, cumpliendo con las metas que este propone.

\section{METODOLOGÍA}

De acuerdo con las finalidades del estudio, para realizar el análisis de los currículos de Estados Unidos, Australia, Singapur y Chile se ha usado el método de análisis de contenido, que es "una técnica de investigación destinada a formular, a partir de ciertos datos, inferencias reproducibles y válidas que puedan aplicarse a su contexto" (Krippenddorff, 1990, p. 28). Esta técnica busca proporcionar 
conocimientos, nuevas interacciones y una representación de los hechos, en nuestro caso, el progreso del álgebra temprana a nivel curricular. Además, permite descubrir la estructura interna de los textos a través del estudio de su contenido semántico (Rico y Fernández-Cano, 2013) y establecer "un conjunto de procedimientos estricto y sistemático para el análisis riguroso, el examen y la verificación de los contenidos de datos escritos" (Cohen, Manion y Morrison, 2011, p. 563).

Para el desarrollo de la investigación se han considerado las siguientes etapas:

1. Seleccionar las unidades de muestreo, en nuestro caso las orientaciones curriculares vigentes de matemáticas de países avanzados en el estudio del álgebra temprana en Educación Infantil y Primaria: Estados Unidos (NCTM, 2003; CCSSM, 2010), Australia (ACARA, 2015), Singapur (NEL, 2013; MOE, 2012) y Chile (MINEDUC, 2012, 2018).

2. Identificar los contenidos u objetivos de aprendizaje que se encuentran vinculados, de manera implícita o explícita, al álgebra temprana, analizando su despliegue y evolución.

3. Organizar los contenidos u objetivos de aprendizaje de álgebra temprana de los distintos currículos, estableciendo categorías inductivas de conocimiento para la Educación Infantil y Primaria. Para llevar a cabo esta organización y establecer dichas categorías se consideran todos los contenidos u objetivos de aprendizaje que aparecen en los distintos currículos analizados, esto supone, por lo tanto, que una categoría no requiera que los contenidos u objetivos de aprendizaje estén presente en todos los currículos. En las tablas 1 y 2 se muestran algunos ejemplos del proceso de obtención de categorías.

Tabla 1. Ejemplificación de la organización de los contenidos curriculares de álgebra temprana y establecimiento de categorías de conocimiento en Educación Infantil

\begin{tabular}{|l|l|l|}
\hline $\begin{array}{c}\text { Unidades de } \\
\text { muestreo }\end{array}$ & \multicolumn{1}{|c|}{ Contenidos } & \multicolumn{1}{c|}{ Categoría de conocimiento } \\
\hline NCTM (2003) & $\begin{array}{l}\text { Seleccionar, clasificar y ordenar } \\
\text { objetos por el tamaño, la cantidad y y } \\
\text { otras propiedades. }\end{array}$ & $\begin{array}{l}\text { Experimentar con elementos u objetos } \\
\text { a partir del reconocimiento de } \\
\text { atributos para establecer relaciones } \\
\text { (clasificaciones, ordenaciones, } \\
\text { correspondencia, etc.) }\end{array}$ \\
\hline NEL (2013) & $\begin{array}{l}\text { Emparejar, clasificar y comparar } \\
\text { cosas por un atributo (por ejemplo } \\
\text { según el color, la forma o tamaño) }\end{array}$ & \\
\hline
\end{tabular}


Tabla 2. Ejemplificación de la organización de los contenidos curriculares de álgebra temprana y establecimiento de categorías de conocimiento en Educación Primaria

\begin{tabular}{|c|l|c|}
\hline $\begin{array}{c}\text { Unidades de } \\
\text { muestreo }\end{array}$ & \multicolumn{1}{|c|}{ Contenidos } & Categoría de conocimiento \\
\hline NCTM (2003) & $\begin{array}{l}\text { Describir y extender patrones } \\
\text { geométricos y numéricos y hacer } \\
\text { generalizaciones acerca de ellos. }\end{array}$ & $\begin{array}{l}\text { Comprensión de distintos tipos } \\
\text { de relaciones y patrones }\end{array}$ \\
\hline ACARA (2015) & $\begin{array}{l}\text { Investigar y describir patrones } \\
\text { numéricos formados por conteo de } \\
\text { saltos y patrones con objetos. }\end{array}$ & \\
\hline
\end{tabular}

4. Establecer un sistema de codificación cuantitativo, por medio de la presencia o ausencia de los contenidos u objetivos de aprendizaje relacionados al álgebra temprana en los diversos currículos analizados, de acuerdo a las categorías inductivas de conocimiento propuestas en la etapa anterior. En concreto, se ha asignado 1 punto en caso de presencia de estos y 0 en caso de ausencia.

5. Sistematizar la información por medio de tablas comparativas y gráficos, cuya lectura permita: a) establecer semejanzas y diferencias a partir del análisis de los contenidos que se promueven en las diversas propuestas curriculares y, b) analizar la presencia de los conocimientos que caracterizan el álgebra temprana en los currículos de Educación Infantil y Primaria.

\section{RESULTADOS}

Considerando las finalidades de nuestro estudio, se describen los datos obtenidos a partir del análisis de contenido de los currículos de Estados Unidos, Australia, Singapur y Chile.

\subsection{El ÁlgeBra temPRANA EN EL CURRÍCULO ESCOLAR DE ESTADOS UNIDOS}

Los documentos oficiales que rigen el currículo escolar de los Estados Unidos son los Principios y Estándares para la Educación Matemática del National Council of Teachers of Mathematics (NCTM, 2003), y los Estándares Comunes para las Matemáticas de la Common Core State Standards for Mathematics (CCSSM, 2010). Resulta de gran interés profundizar en el análisis de estos documentos, dado que 
han tenido un alto impacto a nivel internacional al ser considerados modelos de referencia, generando una repercusión en los currículos de diversos países, sobre todo del contexto latinoamericano como por ejemplo Chile.

Los Principios y Estándares del NCTM (2003) proponen estándares de contenidos y estándares de proceso desde el Prekindergarten hasta el nivel 12 (3-18 años): los estándares de contenido (Números y operaciones, Álgebra, Geometría, Medida, Análisis de datos y probabilidad) describen explícitamente lo que deberían aprender y, los estándares de proceso (resolución de problemas, razonamiento y prueba, conexiones, comunicación y representación) ponen de relieve las formas de adquisición y uso de dichos contenidos. Estos contenidos y procesos "son también reflejo de la cultura matemática que la sociedad necesita, de la práctica educativa anterior, y de los valores y expectativas que comparten los profesores, los educadores matemáticos, los matemáticos y el público en general" (NCTM, 2003, p. xvii).

El Estándar de álgebra "se centra en las relaciones entre cantidades -incluyendo las funciones-, las formas de representación de relaciones matemáticas y el análisis del cambio" (p. 39), como se muestra en la tabla 3.

Los conocimientos algebraicos en la propuesta del NCTM se desarrollan en diversas fases: a) en los primeros niveles se introduce el trabajo con patrones y relaciones, a partir de la manipulación de objetos y posteriormente utilizando secuencias de sonidos y secuencias numéricas; b) así también, se da paso a las representaciones para desarrollar la comprensión de simbología y modelizar situaciones de adicción y sustracción, hasta alcanzar la descripción de cambios tanto cualitativos como cuantitativos; c) luego se fomenta la construcción de patrones numéricos y geométricos y el uso de funciones por medio de tablas y gráficas; d) posteriormente se introduce el concepto de variable como cantidad desconocida y se exploran relaciones mediante ecuaciones; e) finalmente, se introduce la modelización de situaciones usando representaciones de tipo gráfico y tabular para extraer conclusiones, así como el análisis de situaciones de cambios que experimentan dos variables. 
Tabla 3. Estándares de contenido de álgebra temprana para la Educación Infantil y Primaria (NCTM, 2003, p. 402)

\begin{tabular}{|c|c|c|}
\hline & Pre-K-2 (3-8 años) & 3-5 (9-11 años) \\
\hline $\begin{array}{l}\text { Comprender } \\
\text { patrones, } \\
\text { relaciones y } \\
\text { funciones }\end{array}$ & $\begin{array}{l}\text { Seleccionar, clasificar y ordenar objetos } \\
\text { por el tamaño, la cantidad y otras propie- } \\
\text { dades. } \\
\text { Reconocer, describir y ampliar patrones } \\
\text { tales como secuencias de sonidos y for- } \\
\text { mas o sencillos patrones numéricos, y } \\
\text { pasar de una representación a otra. } \\
\text { Analizar cómo se generan patrones de } \\
\text { repetición y de crecimiento. }\end{array}$ & $\begin{array}{l}\text { Describir y extender patrones geomé- } \\
\text { tricos y numéricos y hacer } \\
\text { generalizaciones acerca de ellos. } \\
\text { Representar y analizar patrones y fun- } \\
\text { ciones, verbalmente y mediante tablas } \\
\text { y gráficas. }\end{array}$ \\
\hline $\begin{array}{l}\text { Representar y } \\
\text { analizar } \\
\text { situaciones y } \\
\text { estructuras } \\
\text { matemáticas } \\
\text { utilizando } \\
\text { símbolos } \\
\text { algebraicos }\end{array}$ & $\begin{array}{l}\text { llustrar los principios generales y las pro- } \\
\text { piedades de las operaciones, como la con- } \\
\text { mutatividad, usando números. } \\
\text { Usar representaciones concretas, pictóri- } \\
\text { cas y verbales para desarrollar la com- } \\
\text { prensión de notaciones simbólicas } \\
\text { inventadas y convencionales. }\end{array}$ & $\begin{array}{l}\text { Identificar propiedades como la con- } \\
\text { mutatividad, la asociatividad y distri- } \\
\text { butividad, y emplearlas en el cálculo } \\
\text { con números naturales. } \\
\text { Representar la idea de variable como } \\
\text { cantidad desconocida, por medio de } \\
\text { una letra o un símbolo. } \\
\text { Expresar relaciones matemáticas me- } \\
\text { diante ecuaciones. }\end{array}$ \\
\hline $\begin{array}{l}\text { Utilizar modelos } \\
\text { matemáticos } \\
\text { para } \\
\text { representar y } \\
\text { comprender las } \\
\text { relaciones } \\
\text { cuantitativas } \\
\end{array}$ & $\begin{array}{l}\text { Modelizar situaciones relativas a la adi- } \\
\text { ción y sustracción de números naturales, } \\
\text { utilizando objetos, dibujos y símbolos. }\end{array}$ & $\begin{array}{l}\text { Modelizar situaciones-problema con } \\
\text { objetos, y usar representaciones como } \\
\text { gráficas, tablas y ecuaciones para ex- } \\
\text { traer conclusiones. }\end{array}$ \\
\hline $\begin{array}{l}\text { Analizar el } \\
\text { cambio en } \\
\text { diversos } \\
\text { contextos }\end{array}$ & $\begin{array}{l}\text { Describir cambios cualitativos, como "ser } \\
\text { más alto". } \\
\text { Describir cambios cuantitativos, como el } \\
\text { aumento de estatura de un alumno en } \\
\text { dos pulgadas en un año. }\end{array}$ & $\begin{array}{l}\text { Investigar de qué manera el cambio } \\
\text { que experimenta una variable se rela- } \\
\text { ciona con el de una segunda variable. } \\
\text { Identificar y describir situaciones con } \\
\text { tasas de cambio constantes o varia- } \\
\text { bles, y compararlas. }\end{array}$ \\
\hline
\end{tabular}


Otro referente curricular destacado en Estados Unidos son los CCSSM, que definen "lo que los estudiantes deben entender y poder hacer en su estudio de las matemáticas" (CCSSM, 2010, p.4), y establecen un conjunto de orientaciones que impulsan el desarrollo de una educación de alta calidad. Se organizan en estándares para la práctica matemática y estándares para el contenido matemático. Los estándares para la práctica han sido adaptadas de los cinco estándares de proceso del NCTM (2003) y de los cinco aspectos de competencia descritos en el informe "Adding It Up", del National Research Council de Estados Unidos (NRC, 2001): a) Dar sentido a los problemas; b) Desarrollar un pensamiento abstracto y cuantitativo; c) Construir argumentos viables y criticar el razonamiento de otros; d) Modelar usando matemáticas; e) Usar herramientas adecuadas de manera estratégica; f) Reconocer la importancia de la precisión; g) Buscar y hacer uso de una estructura; y h) Buscar y expresar regularidades en un razonamiento repetido. Los estándares para el contenido matemático responden a una combinación equilibrada de procedimientos y conocimientos que los estudiantes deben manejar para conectar la práctica con el contenido. Para lograr el desarrollo de los estándares de contenido, se establecieron un conjunto de dominios de acuerdo a cada grado educativo: desde Kindergarten hasta el grado 2 (4-8 años) se incluye conteo y cardinalidad, operaciones y pensamiento algebraico, números y operaciones en base diez, medición de datos, geometría; y en los grados 3, 4 y 5 (9-11 años) sus dominios corresponden a operaciones y pensamiento algebraico, números y operaciones en base diez, números y operaciones: fracciones, medición de datos, geometría.

La presencia del álgebra temprana en los estándares de contenido se evidencia en el dominio de operaciones y pensamiento algebraico, como se aprecia en la tabla 4. 
Tabla 4. Estándares de contenidos matemáticos en relación al álgebra temprana en la Educación Infantil y Primaria (CCSSM, 2010)

\begin{tabular}{|c|c|}
\hline & Estándares de contenido \\
\hline $\begin{array}{l}\text { Kindergarten } \\
\text { (3-6 años) }\end{array}$ & Entender la suma como unir y sumar, y entender la resta como separar y tomar de. \\
\hline $\begin{array}{l}\text { Grado } 1 \\
\text { (6-7 años) }\end{array}$ & $\begin{array}{l}\text { Representar y resolver problemas que involucran sumas y restas. } \\
\text { Comprender y aplicar las propiedades de las operaciones y la relación entre la } \\
\text { suma y la resta. } \\
\text { Sumar y restar hasta } 20 . \\
\text { Trabaja con ecuaciones de suma y resta. }\end{array}$ \\
\hline $\begin{array}{l}\text { Grado } 2 \\
\text { (7-8 años) }\end{array}$ & $\begin{array}{l}\text { Representar y resolver problemas que involucran sumas y restas. } \\
\text { Sumar y restar hasta } 20 . \\
\text { Trabaja con grupos iguales de objetos para obtener bases para la multiplicación. }\end{array}$ \\
\hline $\begin{array}{l}\text { Grado } 3 \\
\text { (8-9 años) }\end{array}$ & $\begin{array}{l}\text { Representar y resolver problemas de multiplicación y división. } \\
\text { Comprender las propiedades de la multiplicación y la relación entre } \\
\text { multiplicación y división. } \\
\text { Multiplica y divide entre } 100 \text {. } \\
\text { Resuelve problemas relacionados con las cuatro operaciones e identifique y } \\
\text { explica patrones en aritmética. }\end{array}$ \\
\hline $\begin{array}{l}\text { Grado } 4 \\
\text { (9-10 años) }\end{array}$ & $\begin{array}{l}\text { Usa las cuatro operaciones con números enteros para resolver problemas. } \\
\text { Familiarizarse con factores y múltiplos. } \\
\text { Generar y analizar patrones. }\end{array}$ \\
\hline $\begin{array}{l}\text { Grado } 5 \\
\text { (10-11 años) }\end{array}$ & $\begin{array}{l}\text { Escribir e interpretar expresiones numéricas. } \\
\text { Analizar patrones y relaciones. }\end{array}$ \\
\hline
\end{tabular}

El pensamiento algebraico en los CCSSM (2010) se desarrolla por medio de las siguientes fases: a) los primeros grados se encuentran relacionados fuertemente con la operatoria de adición y sustracción, al profundizar en términos como unir y separar, representar y comprender sus propiedades e incorporar trabajos con ecuaciones de sumas y restas; b) luego se establece la operatoria de multiplicación y división (Grado 3), por medio de la resolución de problemas, la comprensión de sus propiedades y la relación entre ambas operaciones. A partir de las cuatro operaciones básicas se introduce al trabajo con patrones numéricos; c) finalmente se establecen vínculos entre la operatoria y las expresiones numéricas, dando origen a la generalización y al análisis de patrones y relaciones durante los Grados 4 y 5. 
A modo general, se puede apreciar que el álgebra temprana está presente en los CCSSM (2010) desde los primeros grados educativos al igual que en el NCTM (2003); sin embargo, éstos últimos resultan ser más coherentes con el estudio del álgebra. Se observan también algunas diferencias en la evolución y propuesta para el desarrollo de ciertos contenidos, como lo es la generación de patrones y relaciones, que se evidencian en grados más avanzados según los CCSSM, mientras que el NCTM los promueve desde edades tempranas.

\subsection{El Álgebra temprana en el CURRículo escolar de Australia}

El currículo de matemáticas australiano, que depende del organismo Australian Curriculum, Assessment and Reporting Authority (ACARA), se enfoca en "desarrollar una comprensión matemática sofisticada y refinada, con fluidez, razonamiento lógico, pensamiento analítico y habilidades para resolver problemas" (ACARA, 2015, p. 4), comenzando su desarrollo desde Foundation (5 años) hasta el año 12 (18 años). Se estructura en tres líneas de contenido (Números y álgebra, Medición y geometría, Estadística y probabilidad) y cuatro de competencias (comprensión, fluidez, resolución de problemas, razonamiento). Las líneas de competencia describen las acciones en las que los estudiantes pueden participar al aprender y usar el contenido, proporcionando el lenguaje para construir en el desarrollo del aprendizaje de las matemáticas. Este currículo, además, garantiza el vínculo entre las matemáticas y otras disciplinas, es decir, propicia que las diversas interrelaciones que componen la matemática, tales como, habilidades, conceptos, procesos, propiedades, etc., puedan ser aplicadas más allá del aula de matemáticas.

Los contenidos que se abordan en la línea de Números y Álgebra se agrupan en seis sublíneas de contenido, que se desarrollan de acuerdo al nivel educativo de los estudiantes: Números y valor posicional (F-8), Fracciones y decimales (1-6), Números reales (7-10), Dinero y matemáticas financieras (1-10), Patrones y álgebra (F-10), relaciones lineales y no lineales (7-10). Los contenidos correspondientes a la sublínea de Patrones y álgebra se presentan en la tabla 5. 
Tabla 5. Descripciones de contenido de la sublínea "Patrones y álgebra" para la Educación Infantil y Primaria (ACARA, 2015)

\begin{tabular}{|c|c|}
\hline & Contenidos \\
\hline $\begin{array}{l}\text { Foundation } \\
\text { (5-6 años) }\end{array}$ & $\begin{array}{l}\text { Ordenar y clasificar objetos familiares y explicar la base de estas clasificaciones. } \\
\text { Copiar, continuar y crear patrones con objetos y dibujos. }\end{array}$ \\
\hline $\begin{array}{l}\text { Año } 1 \\
(6-7 \text { años) } \\
\end{array}$ & $\begin{array}{l}\text { Investigar y describir patrones numéricos formados por conteo de saltos y patro- } \\
\text { nes con objetos. }\end{array}$ \\
\hline $\begin{array}{l}\text { Año } 2 \\
\text { (7-8 años) }\end{array}$ & $\begin{array}{l}\text { Describir patrones numéricos e identificar elementos faltantes. } \\
\text { Resolver problemas usando oraciones numéricas para sumar o restar. }\end{array}$ \\
\hline $\begin{array}{l}\text { Año } 3 \\
(8-9 \text { años) }\end{array}$ & $\begin{array}{l}\text { Describir, continuar y crear patrones numéricos resultantes de realizar sumas o } \\
\text { restas. }\end{array}$ \\
\hline $\begin{array}{l}\text { Año } 4 \\
\text { (9-10 años) }\end{array}$ & $\begin{array}{l}\text { Explorar y describir patrones numéricos resultantes de realizar la multiplicación. } \\
\text { Resolver problemas verbales mediante el uso de oraciones numéricas que impli- } \\
\text { quen multiplicación y división donde no hay resto. } \\
\text { Encontrar cantidades desconocidas en oraciones numéricas que involucran suma } \\
\text { y resta e identificar oraciones numéricas equivalentes que involucren suma o resta. }\end{array}$ \\
\hline $\begin{array}{l}\text { Año } 5 \\
(10-11 \text { años) }\end{array}$ & $\begin{array}{l}\text { Describir, continuar y crear patrones con fracciones, decimales y números enteros } \\
\text { resultantes de realizar sumas y restas. } \\
\text { Encontrar cantidades desconocidas en oraciones numéricas que involucren mul- } \\
\text { tiplicación y división e identificar oraciones numéricas equivalentes que involu- } \\
\text { cren multiplicación y división. }\end{array}$ \\
\hline $\begin{array}{l}\text { Año } 6 \\
(11-12 \text { años) }\end{array}$ & $\begin{array}{l}\text { Continuar y crear secuencias que involucren números enteros, fracciones y deci- } \\
\text { males. Describir la regla utilizada para crear la secuencia. } \\
\text { Explorar el uso de corchetes y orden de las operaciones para escribir oraciones } \\
\text { numéricas. }\end{array}$ \\
\hline
\end{tabular}

Los conocimientos que promueve el currículo australiano para alcanzar el desarrollo del pensamiento algebraico están presentes desde la Educación Infantil (Foundation) y se desarrollan por medio de las siguientes fases: a) inicialmente con la clasificación de objetos y luego en el trabajo con patrones, desde su forma experiencial, usando materiales, sonidos, movimientos o dibujos; b) luego, al iniciar la Educación Primaria, se profundiza el trabajo con patrones, al describir patrones numéricos y establecer relaciones. El uso de patrones numéricos se extiende y profundiza en toda la Educación Primaria; c) en los niveles posteriores, los estudiantes deben crear patrones numéricos como resultado de realizar operaciones de adición, sustracción, multiplicación y división, hasta alcanzar la 
descripción de patrones con fracciones, decimales y números enteros; y d) al finalizar la Educación Primaria, los estudiantes deben ser capaces de crear secuencias numéricas que involucren números enteros y racionales, explicando la regla utilizada. Por otro lado, se evidencia la resolución de problemas al utilizar números en diversos contextos que impliquen operatoria de adición, sustracción, multiplicación y divisiones exactas. Con el desarrollo de este contenido, el currículo realiza una primera aproximación al trabajo con ecuaciones y desigualdades, al encontrar cantidades desconocidas en oraciones numéricas e identificar equivalencias. Finalmente, se explora en el uso de paréntesis y prioridad de las operaciones al escribir oraciones numéricas.

Así pues, para alcanzar el desarrollo del pensamiento algebraico, tanto el currículo de Estados Unidos como el de Australia, proponen el trabajo con patrones desde la Educación Infantil. Para ello, ambos currículos acercan este contenido a la experiencia de los estudiantes, por medio del reconocimiento y descripción de patrones en secuencias de sonido, formas, objetos y dibujos.

Por otra parte, a diferencia del currículo estadounidense, el currículo australiano aborda una cantidad menor de contenidos durante la Educación Primaria, pero profundiza ampliamente en el trabajo con patrones y secuencias. Así también, se da prioridad al trabajo con operaciones numéricas, uso de incógnitas y expresiones equivalentes.

\subsection{El ÁlgEBra temprana en EL CURRÍCULO ESCOLAR DE SingaPUR}

El sistema educativo en Singapur se rige por el Ministerio de Educación (MOE, Ministry of Education) y comienza a implementarse en la Educación Infantil (4 a 6 años de edad). El desarrollo de la Educación Infantil se fundamenta a partir del marco Nurturing Early Learners (NEL), en el que el ámbito de las matemáticas se establece a partir del área de aprendizaje de la Aritmética. Según el marco NEL (2013, p. 6), "el desarrollo de conceptos y habilidades de aritmética, implica ayudar a los niños a conocer y usar conceptos y habilidades, de modo que establezcan relaciones y conexiones que luego puedan aplicar significativamente en sus experiencias diarias". En este sentido, la Aritmética como área de aprendizaje se desarrolla en la Educación Infantil a través de tres líneas centrales: Relaciones y patrones simples, Conteo y sentido numérico, Formas básicas y conceptos espaciales simples. 
El estudio del álgebra temprana se ve incorporado explícitamente en la línea de Relaciones y patrones, donde se conocen y establecen relaciones simples a través del desarrollo de habilidades y conceptos, tales como emparejar, clasificar, comparar y ordenar. Así también, el trabajo con patrones permite a los niños desarrollar sus capacidades de pensamiento lógico, las cuales son fundamentales para comprender el concepto de número (NEL, 2013).

El plan de estudio de matemáticas de Educación Primaria pone especial énfasis en cinco componentes (comprensión conceptual, dominio de habilidades, procesos matemáticos, metacognición y actitudes) y está organizado en tres líneas de contenido: Números y álgebra, Medición y geometría, Estadística. La línea de contenido de Números y álgebra se divide en sublíneas de acuerdo a cada nivel educativo (Números enteros, Dinero, Fracciones, Decimales, Porcentajes, Relaciones proporcionales, Tasa y velocidad). Los contenidos de álgebra que se evidencian a lo largo del currículo (tabla 6) están ligados principalmente a la sublínea de contenido de Números Enteros, sin embargo, en el nivel de Primaria 6 se presenta una sublínea específica para el estudio del álgebra temprana.

Tabla 6. Contenidos en relación al estudio del álgebra temprana en currículo de Singapur de Educación Infantil (NEL, 2013) y Educación Primaria (Ministry of Education Singapore, 2012)

\begin{tabular}{|c|c|}
\hline & Contenidos \\
\hline $\begin{array}{l}\text { Kindergarten } \\
\text { (4-6 años) }\end{array}$ & $\begin{array}{l}\text { Emparejar, clasificar y comparar cosas por un atributo (por ejemplo } \\
\text { según el color, la forma o tamaño) } \\
\text { Poner las cosas en orden según el tamaño o la longitud y eventos de secuencia } \\
\text { Reconocer, extender y crear patrones simples (por ejemplo: patrón AB) }\end{array}$ \\
\hline $\begin{array}{l}\text { Primaria } 1 \\
\text { (6-7 años) }\end{array}$ & $\begin{array}{l}\text { Números enteros: Números hasta el } 100 \\
\text { Comparar y ordenar números } \\
\text { Patrones en secuencias numéricas } \\
\text { Números ordinales (primero, segundo hasta el décimo) y símbolos }\left(1^{\circ}, 2^{\circ}, 3^{\circ} \text {, etc.) }\right.\end{array}$ \\
\hline $\begin{array}{l}\text { Primaria } 2 \\
\text { (7-8 años) }\end{array}$ & $\begin{array}{l}\text { Números enteros: Números hasta el } 1000 \\
\text { Comparar y ordenar números } \\
\text { Patrones en secuencias numéricas } \\
\text { Números pares e impares }\end{array}$ \\
\hline $\begin{array}{l}\text { Primaria } 3 \\
\text { (8-9 años) }\end{array}$ & $\begin{array}{l}\text { Números enteros: Números hasta } 10.000 \\
\text { Comparar y Ordenar números } \\
\text { Patrones en secuencias numéricas }\end{array}$ \\
\hline
\end{tabular}




\begin{tabular}{ll}
\hline Primaria 4 & Números enteros: Números hasta el 100.000 \\
$(9-10$ años $)$ & Comparar y ordenar números \\
& Patrones en secuencias numéricas \\
\hline Primaria 5 & Relaciones: \\
$(10-11$ años) & Notación, representación e interpretación de $a: b$ y a: $b: c$, donde $a, b$ y c son \\
& números enteros, excluyendo las relaciones que implican fracciones y decimales \\
& Relaciones equivalentes \\
& Dividir una cantidad en una relación determinada \\
& Expresar una relación en su forma más simple \\
& Encontrar la proporción de dos o tres cantidades dadas \\
& Encontrar el término que falta en un par de relaciones equivalentes \\
& Resolver problemas de palabras en dos pasos en relación con la proporción \\
\hline Primaria 6 & Álgebra: \\
Uso de letras para representar un número desconocido \\
& Notación, representación e interpretación de expresiones algebraicas simples, \\
& tales como: $a \pm 3, a \times 3$ ó $3 a$ años 3 a 3 \\
& Simplificar expresiones lineales simples excluyendo los paréntesis \\
& Evaluar expresiones lineales simples por sustitución \\
& Resolver ecuaciones lineales simples que implican un coeficiente de números \\
& enteros sólo en contextos simples
\end{tabular}

La presencia del álgebra temprana en las orientaciones curriculares de Singapur se manifiesta en las siguientes etapas: a) la Educación Infantil se ve marcada por el reconocimiento de patrones simples y relaciones de números por medio de la clasificación, orden y comparación de objetos, b) mientras tanto, en la Educación Primaria el desarrollo curricular de estos contenidos se vincula al estudio de los Números enteros. Los Números enteros se trabajan de forma gradual, partiendo desde el 100 en Primaria 1 hasta el 10.000 en Primaria 4. Así pues, se establece en cada uno de estos niveles la comparación y orden de estos números, dando paso a la descripción y creación de patrones a partir del análisis de las secuencias numéricas que se registran; c) por otra parte, en Primaria 5, los contenidos relacionados con el álgebra temprana se manifiestan a través de la sublínea de Relaciones, haciendo uso de la notación algebraica para representar el concepto de razón, estableciendo relaciones equivalentes para introducir el concepto de proporción, y con ello determinar el término desconocido en relaciones equivalentes; d) luego, en el nivel de Primaria 6, se dedica de forma preferente una sublínea de contenido al estudio del álgebra temprana por medio del lenguaje algebraico al emplear letras para representar una cantidad desconocida, y traducir a números 
y símbolos expresiones del lenguaje común. Así también, se origina el uso de expresiones lineales simples al evaluar expresiones algebraicas por sustitución y reducir términos semejantes por medio de la simplificación. El desarrollo del pensamiento algebraico finaliza en Educación Primaria con la resolución de ecuaciones lineales de primer grado en contextos simples.

A modo general, los contenidos que promueve el currículo de Singapur para alcanzar el desarrollo del pensamiento algebraico desde las primeras edades se fundamentan, en gran medida, en el uso de patrones y sucesiones numéricas, en sintonía con los currículos de Estados Unidos y Australia.

Por otro lado, tanto las orientaciones curriculares de Singapur como de Australia, vinculan el estudio del álgebra temprana a los números, al ser considerados como parte de una sola línea de contenidos. Ambos currículos destacan la importancia de trabajar estos contenidos en forma simultánea, dado que uno complementa el estudio del otro, es decir, la enseñanza de los números requiere del álgebra y el estudio del álgebra requiere de la comprensión de los números.

\subsection{El ÁlgeBra temprana en El CURRículo escolar DE CHILE}

En Chile, el Ministerio de Educación (MINEDUC) plantea el currículo para el desarrollo de la Educación Infantil a partir de las Bases Curriculares para la Educación Parvularia (MINEDUC, 2018), las cuales constituyen un referente a nivel nacional donde se "define principalmente qué y para qué deben aprender los párvulos desde sus primeros meses de vida hasta el ingreso de la Educación Básica" (p. 9).

Los objetivos de aprendizaje que proponen estas Bases Curriculares se organizan en tres ámbitos de experiencia: Desarrollo personal y social, Comunicación integral, Interacción y comprensión del entorno. Este último ámbito de aprendizaje alberga el núcleo Pensamiento Matemático, haciendo referencia a los diferentes procesos a través de los cuales los niños interpretan y explican los diversos elementos y situaciones del entorno, tales como, ubicación en el espacio-tiempo, relaciones de orden, comparación, clasificación, seriación, identificación de patrones, etc., como también, a la construcción de la noción de número y el uso inicial de la función ordenadora y cuantificadora del mismo.

Para efectos de este estudio, nos centraremos en los objetivos curriculares relacionados con la enseñanza del álgebra temprana correspondientes al $3^{\circ}$ nivel curricular, nivel de Transición (tabla 7), referido a niños de 4 a 6 años de edad. 
Por otra parte, la implementación del currículo escolar para la Educación Básica (6 a 12 años) se fundamenta a partir de las Bases Curriculares Primero a Sexto Básico (MINEDUC, 2012). La asignatura de matemáticas tiene como propósito "enriquecer la comprensión de la realidad, facilitar la selección de estrategias para resolver problemas y contribuir al desarrollo del pensamiento crítico y autónomo en todos los estudiantes" (MINEDUC, 2012, p. 214). Para desarrollar el pensamiento matemático se establecen cuatro habilidades relacionadas entre sí: resolver problemas, argumentar y comunicar, modelar y representar. Estas habilidades representan un rol fundamental en la adquisición de nuevas destrezas, conceptos y en la aplicación de conocimientos para resolver problemas rutinarios y no rutinarios, propios de la matemática y de otros ámbitos. Así también, la adquisición de nuevos conceptos en la asignatura de matemáticas se organiza a través de cinco ejes temáticos: Números y operaciones, Patrones y álgebra, Geometría, Medición, Datos y probabilidades.

El eje de patrones y álgebra pretende que los estudiantes expliquen y describan relaciones de todo tipo, entre números, formas, objetos y conceptos. El uso de patrones, observados en secuencia de objetos, imágenes o números que representan regularidades, debe ser representado de manera concreta, pictórica y simbólica, desde donde los estudiantes deben ser capaces de transitar de una forma de representación a otra.

La tabla 7 muestra los objetivos de aprendizaje vinculados al álgebra temprana que plantean las Bases Curriculares del Ministerio de Educación para la Educación Parvularia y Educación Básica.

Tabla 7. Objetivos de aprendizaje en relación al álgebra temprana en la Educación Parvularia (MINEDUC, 2018) y Educación Básica (MINEDUC, 2012)

\begin{tabular}{ll}
\hline & Objetivos de Aprendizaje \\
\hline $3^{\circ}$ Nivel de & Crear patrones sonoros, visuales, gestuales, corporales u otros, de dos o tres ele- \\
Transición & mentos. \\
(4-6 años) & Experimentar con diversos objetos estableciendo relaciones al clasificar por dos o \\
& tres atributos a la vez (forma, color, tamaño, función, masa, materialidad, entre \\
& otros) y seriar por altura, ancho, longitud o capacidad para contener. \\
& Emplear cuantificadores, tales como: "más que", "menos que", "igual que", al com- \\
& parar cantidades de objetos en situaciones cotidianas. \\
\hline
\end{tabular}


$1^{\circ}$ Básico Reconocer, describir, crear y continuar patrones repetitivos (sonidos, figuras, ritmos...) y (6-7 años) patrones numéricos hasta el 20, crecientes y decrecientes, usando material concreto, pictórico y simbólico, de manera manual y/o por medio de software educativo.

Describir y registrar la igualdad y la desigualdad como equilibrio y desequilibrio, usando una balanza en forma concreta, pictórica y simbólica del 0 al 20, usando el símbolo igual (=).

$2^{\circ}$ básico Crear, representar y continuar una variedad de patrones numéricos y completar

(7-8 años) los elementos faltantes, de manera manual y/o usando software educativo.

Demostrar, explicar y registrar la igualdad y la desigualdad en forma concreta y pictórica del 0 al 20, usando el símbolo igual (=) y los símbolos no igual $(>,<)$.

$3^{\circ}$ Básico Generar, describir y registrar patrones numéricos, usando una variedad de estra(8-9 años) tegias en tablas del 100, de manera manual y/o con software educativo.

Resolver ecuaciones de un paso que involucren adiciones y sustracciones y un símbolo geométrico que represente un número desconocido, en forma pictórica y simbólica del 0 al 100.

$4^{\circ}$ Básico Identificar y describir patrones numéricos en tablas que involucren una opera(9-10 años) ción, de manera manual y/o usando software educativo.

Resolver ecuaciones e inecuaciones de un paso que involucren adiciones y sustracciones, comprobando los resultados en forma pictórica y simbólica del 0 al 100 y aplicando las relaciones inversas entre la adición y la sustracción.

$5^{\circ}$ Básico Descubrir alguna regla que explique una sucesión dada y que permita hacer (10-11 años) predicciones.

Resolver problemas, usando ecuaciones e inecuaciones de un paso, que involucren adiciones y sustracciones, en forma pictórica y simbólica.

$6^{\circ}$ Básico Demostrar que comprenden la relación entre los valores de una tabla y aplicarla (11-12 años) en la resolución de problemas sencillos:

a) identificando patrones entre los valores de la tabla

b) formulando una regla con lenguaje matemático

Representar generalizaciones de relaciones entre números naturales, usando expresiones con letras y ecuaciones.

Resolver ecuaciones de primer grado con una incógnita, utilizando estrategias como:

a) usar una balanza

b) usar la descomposición y la correspondencia 1 a 1 entre los términos en cada lado de la ecuación y aplicando procedimientos formales de resolución.

El álgebra temprana está presente en todos los niveles del currículo escolar chileno. La adquisición del aprendizaje del álgebra en los estudiantes se evidencia a partir del progreso de diversas etapas: a) comenzando en el nivel de transición para la Educación Parvularia con la indagación en diversos patrones (sonoros, visuales, gestuales, etc.) y, estableciendo relaciones de clasificación y 
cuantificación a partir de diversos objetos; b) posteriormente en los primeros niveles de la Educación Básica se inicia el desarrollo de un trabajo más amplio con reconocimiento y descripción de patrones repetitivos, para avanzar hacia el registro de patrones numéricos de forma manual y/o software educativos por medio de tablas; c) para luego descubrir y formular reglas con lenguaje matemático a partir de una sucesión para realizar predicciones e indagar en el trabajo con ecuaciones e inecuaciones que involucre adiciones y sustracciones; d) hasta finalizar la Educación Básica con la resolución de ecuaciones de primer grado con una incógnita, aplicando procedimientos formales.

Si bien, el tratamiento que recibe el álgebra en el currículo escolar chilenos para la Educación Infantil y Primaria se ve influenciado fuertemente por las directrices que proponen los Principles and Standard for School Mathematics (NCTM, 2003), no se ha considerado abordar de manera más específica la modelización de problemas utilizando tablas, gráficas y ecuaciones y, el análisis de situaciones de cambio por medio del uso de variables.

\section{5 ¿QUÉ CONOCIMIENTOS PROMUEVE EL CURRÍCULO ESCOLAR PARA DESARROLLAR EL PENSAMIENTO ALGEBRAICO? HACIA UNA CARACTERIZACIÓN DEL ÁLGEBRA TEMPRANA}

A partir del análisis de las diversas propuestas curriculares podemos evidenciar el despliegue y evolución de los conocimientos que se ven implicados en el estudio del álgebra temprana de algunos currículos contemporáneos de Educación Infantil y Primaria que incluyen explícitamente conocimientos de esta naturaleza. Para alcanzar el desarrollo del pensamiento algebraico, estos currículos proponen abordar en cada nivel educativo diversos conocimientos por medio de la adquisición de conceptos y habilidades. El bloque de álgebra temprana impulsa el tratamiento de diversos contenidos, estableciendo ciertas diferencias en el grado de profundización y prioridad que estos consiguen a lo largo del currículo escolar.

En relación a la Educación Infantil, y con base a poder caracterizar el álgebra temprana y analizar su presencia o ausencia en los currículos de esta etapa, se han establecido tres categorías de contenidos a partir de la revisión curricular realizada:

- Experimentar con elementos u objetos a partir del reconocimiento de atributos para establecer relaciones (clasificaciones, ordenaciones, correspondencia, etc.). 
- Seriación a partir de patrones de repetición: Identificación, construcción y representación del patrón.

- Descripción de cambios cualitativos y cuantitativos.

A partir de éstas categorías, hemos clasificado los contenidos propuestos en las orientaciones curriculares. La figura 1, muestra las diferencias respecto a la presencia de los conocimientos que caracterizan el álgebra temprana en la Educación Infantil en los currículos contemporáneos considerados en este estudio.

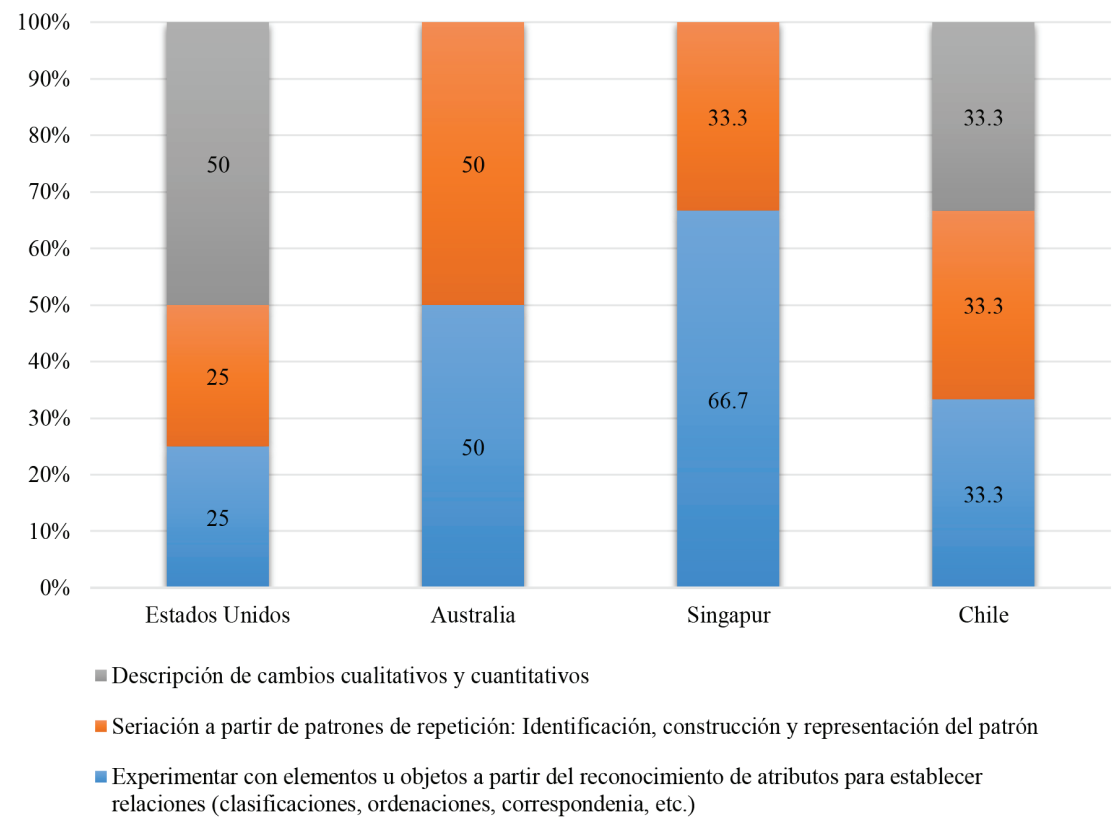

Figura 1. Presencia de los conocimientos que caracterizan el álgebra temprana en la Educación Infantil.

El conocimiento relacionado a la experimentación con elementos u objetos a partir del reconocimiento de atributos para establecer relaciones se evidencia en todos los currículos contemporáneos, alcanzando una mayor presencia (por encima del 65\%) en el currículo de Singapur. Una menor presencia presenta el conocimiento correspondiente a la descripción de cambios cualitativos y cuantitativos (no supera el 50\%), dado que sólo se observa en los currículos de 
Estados Unidos y Chile, siendo el conocimiento con mayor predominancia para el currículo de Estados Unidos, quedando ausente en los currículos de Australia y Singapur.

El conocimiento correspondiente a seriación a partir de patrones de repetición está presente en todas las orientaciones curriculares, pero su presencia no excede el 50\%.

Cabe señalar que el currículo australiano muestra un equilibrio en la presencia de los conocimientos que promueve el currículo para el desarrollo del álgebra temprana en la Educación Infantil, considerando la experimentación con elementos para establecer relaciones y la seriación a partir de patrones. A su vez en el currículo chileno, la presencia de los conocimientos que se establecen para alcanzar el pensamiento algebraico en la Educación Infantil se desarrolla de manera proporcional.

Por otra parte, para realizar el análisis comparativo de los currículos de Educación Primaria se han considerado las categorías de conocimientos importantes de álgebra temprana para esta etapa propuestos por Alsina (2019):

- La comprensión de distintos tipos de relaciones (de equivalencia, de orden, etc.) y de patrones (de crecimiento, de decrecimiento, etc.).

- El uso de símbolos algebraicos y modelos matemáticos para representar situaciones matemáticas.

- La comprensión del cambio.

- Además, a partir del análisis de las orientaciones curriculares se ha incorporado otra categoría: el uso de variables para determinar una constante o incógnita, dado que al revisar los contenidos que promueven las orientaciones curriculares, especialmente en los últimos niveles de Educación Primaria, se plantea iniciar la comprensión conceptual respecto de los diferentes usos de la variable como cantidad desconocida, ya sea para determinar el término que falta en un par de relaciones equivalentes, determinar una cantidad desconocida en oraciones numéricas, determinar una proporción, resolver ecuaciones lineales e inecuaciones, entre otros.

A partir de las categorías de conocimiento propuestas, hemos clasificado los contenidos revisados en las orientaciones curriculares. La figura 2, muestra las diferencias relacionadas con la presencia de los conocimientos que caracterizan el álgebra temprana en la Educación Primaria en los currículos de los diferentes países analizados en este estudio. 


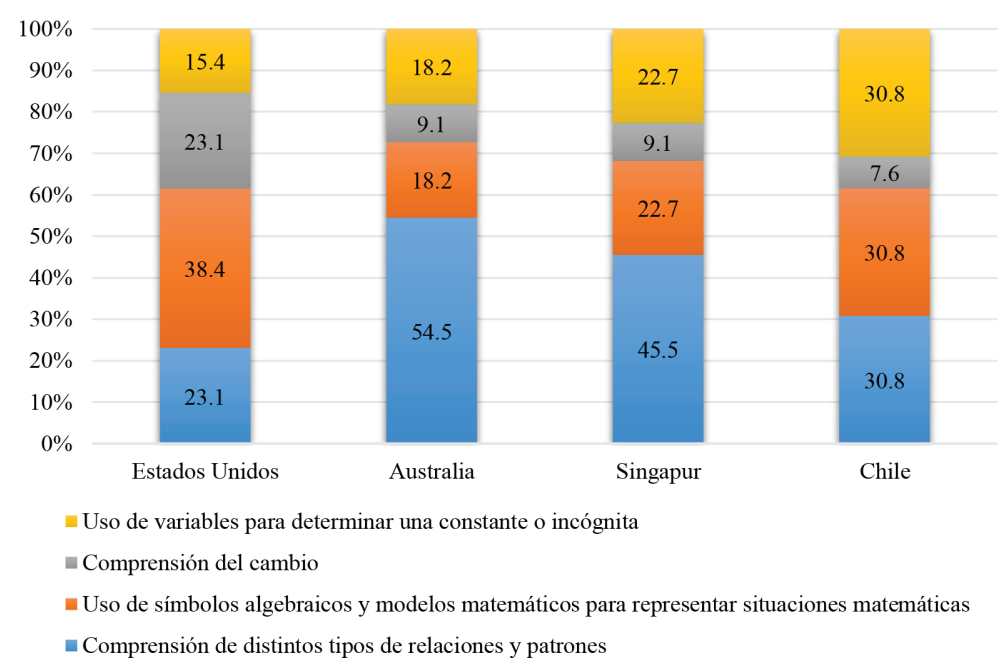

Figura 2. Presencia de los conocimientos que caracterizan el álgebra temprana en la Educación Primaria.

Los resultados obtenidos muestran una mayor presencia del conocimiento vinculado a la comprensión de distintos tipos de relaciones y patrones en los currículos de Australia y Singapur (por encima del 45\%). En el currículo de Estados Unidos, la mayor presencia de los conocimientos se relaciona al uso de símbolos algebraicos y modelos matemáticos para representar situaciones (alcanzando 38,4\%). En el caso de Chile, tres de los cuatro conocimientos que caracterizan el álgebra temprana en la Educación Primaria se desarrollan en forma proporcional a lo largo del currículo escolar, con una presencia del 30,8\%. Finalmente, se observa una presencia menor del conocimiento relacionado a la comprensión del cambio (por debajo del 25\%) en los currículos de Estados Unidos, Australia, Singapur y Chile.

La revisión de los currículos contemporáneos nos ha permitido realizar una primera aproximación hacia la caracterización del álgebra temprana. A partir de este análisis y considerando los referentes teóricos estudiados, realizamos una primera caracterización del álgebra temprana como la capacidad de desarrollar modos de pensamiento algebraico durante las primeras edades en situaciones vinculadas tanto al álgebra propiamente como a otras áreas del currículo de matemática, tales como números, geometría, medida, etc. Para empoderar estos modos de pensamiento algebraico, se debería capacitar a todos los niños y niñas de Educación Infantil para experimentar con elementos u objetos a partir del 
reconocimiento de atributos con el propósito de establecer relaciones (clasificaciones, ordenaciones, correspondencia, etc.), realizar seriaciones a partir de patrones de repetición (identificación, construcción y representación del patrón) y describir cambios cualitativos y cuantitativos; y se debería capacitar a todos los niños y niñas de Educación Primaria para comprender distintos tipos de relaciones (de equivalencia, de orden, etc.) y de patrones (de crecimiento, de decrecimiento, etc.), usar símbolos algebraicos y modelos matemáticos para representar situaciones matemáticas, comprender el cambio y usar variables para determinar una constante o incógnita.

\section{CONSIDERACIONES FINALES}

En este artículo se ha presentado un análisis de la incorporación del álgebra temprana en las etapas de Educación Infantil y Primaria (4 a 12 años de edad aproximadamente) a nivel curricular. Para ello, se han analizado las orientaciones curriculares de países que incluyen explícitamente este bloque de contenido desde los primeros años de escolaridad. Esta revisión ha permitido, por una parte, caracterizar el álgebra temprana a partir de los conocimientos que fomentan el desarrollo del pensamiento algebraico en Educación Infantil y Primaria y, por otra, realizar un estudio comparativo que evidencie la presencia de estos conocimientos, a modo de itinerario vertical a lo largo de todo el currículo escolar.

A modo de síntesis, los conocimientos que caracterizan el álgebra temprana en Educación Infantil son: experimentación con elementos a partir del reconocimiento de atributos para establecer relaciones (clasificaciones, ordenaciones, correspondencias, etc.); seriaciones a partir de patrones de repetición (identificación, construcción y representación del patrón); y descripción de cambios cualitativos y cuantitativos y; en Educación Primaria: comprensión de distintos tipos de relaciones (de equivalencia, de orden, etc.) y de patrones (de crecimiento, de decrecimiento, etc.); uso de símbolos algebraicos y modelos matemáticos para representar situaciones matemáticas; comprensión del cambio; y uso de variables para determinar una constante o incógnita.

Por otra parte, los resultados obtenidos muestran que el desarrollo del pensamiento algebraico se encuentra estrechamente relacionado con el tratamiento y evolución que reciben los contenidos matemáticos vinculados a la enseñanza del álgebra en los distintos niveles educativos. En este sentido, el estudio del álgebra temprana en la Educación Infantil se ve marcada por la experimentación con 
elementos u objetos a partir del reconocimiento de atributos para establecer relaciones, con una presencia media del $43,8 \%$, mientras que el conocimiento con menor presencia corresponde a la descripción de cambios cualitativos y cuantitativos, con una presencia media del 20,8\%. En la Educación Primaria se profundiza mayoritariamente en la comprensión de distintos tipos de relaciones y patrones, teniendo una presencia media del 38,5\%, mientras que el conocimiento con menor presencia es la comprensión del cambio, con una presencia media del $12,2 \%$.

Todos los currículos que hemos analizado enfatizan la importancia del desarrollo de los patrones y relaciones como base para construir un itinerario algebraico desde la Educación Infantil a Primaria. Por ello, es necesario establecer una estrecha coordinación entre ambas etapas educativas para garantizar la continuidad de los objetivos establecidos en las orientaciones curriculares y lograr una enseñanza eficaz del álgebra temprana desde las primeras edades, que asegure las bases para su posterior profundización en la educación secundaria.

Así también, dado los cambios que han sufrido los planes curriculares escolares durante los últimos años, consideramos que es necesario ofrecer a los futuros maestros y a los maestros en activo experiencias de formación que incorporen aspectos que permitan reconocer y promover el desarrollo del pensamiento algebraico en sus estudiantes. Estas experiencias de formación deben suponer, por una parte, el desarrollo y reflexión sobre tareas matemáticas de carácter algebraico, así como estrategias para propiciar su enseñanza, mediante la representación de situaciones y problemas con patrones, secuencias, uso de expresiones simbólicas formuladas con variables, modelización, funciones, proporcionalidad, expresiones equivalentes, planteamiento de fórmulas sencillas y resolución de ecuaciones.

\section{AGRADECIMIENTOS}

Este trabajo fue apoyado por la Agencia Nacional de Investigación y Desarrollo del Gobierno de Chile (ANID) mediante una beca de doctorado en el extranjero, Folio $N^{\circ} 72200447$. 


\section{REFERENCIAS}

ACARA (2015). The Australian Curriculum: Mathematics. Recuperado de: https://www. australiancurriculum.edu.au/f-10-curriculum/mathematics/

Alsina, Á. (2019). Itinerarios didácticos para la enseñanza de las matemáticas (6-12 años). Graó. Andréu, J. (2002). Las técnicas de Análisis de Contenido: Una revisión actualizada. Editorial Fundación Centro de Estudios Andaluces.

Ball, D.L., Thames, M.H. y Phelp, G. (2008). Content Knowledge for Teaching: What Makes it Special? Journal of Teacher Education, 59(5), 389-407.

Bastable, V y Schifter, D. (2007). Classroom stories: examples of elementary students engaged in Early Algebra. En J. Kaput, D. W. Carraher y M. L. Blanton (Eds.), Algebra in the Early Grades (pp. 165-184). Lawrence Erlbaum Associates.

Blanton M. L., y Kaput J. (2005). Characterizing a classroom practice that promotes algebraic reasoning. Journal for Research in Mathematics Education, 36(5), 412-446.

Carpenter, T. P., Franke, M. L. y Levi, L. (2003). Thinking mathematically: Integrating arithmetic y algebra in elementary school. Heinemann.

Carraher, D. W. y Schliemann, A. D. (2007). Early algebra and algebraic reasoning. En F. K. Lester (Ed.), Second handbook of research on mathematics teaching and learning (pp. 669-705). NCTM e IAP.

Carraher, D. W. y Schliemann, A. D. (2019). Early algebraic thinking and the US mathematics standards for grades K to 5. Estudio de Educación y Desarrollo, 42(3), 479-522

Carraher, D. W., Schliemann, A. D. y Brizuela, B. M. (2000). Early algebra, early arithmetic: Treating operations as functions. Presentado en the Twenty-second annual meeting of the North American Chapter of the International Group for the Psychology of Mathematics Education, Tucson, Arizona.

Cohen, L., Manion, L. y Morrison, K. (2011). Research methods in education. Routledge. Common Core State Standars for Mathematics (2010). Common Core State Standards Initiative. http://www.corestandards.org/wp-content/uploads/Math_Standards1.pdf

Davis, R. B. (1985). ICME-5 Report: Algebraic thinking in the early grades. Journal of Mathematical Behaviour, 4, 195-208.

Hill H. C., Ball D.L. y Schilling S.G. (2008): Unpacking pedagogical content knowledge: Conceptualizing and measuring teachers' topic-specific knowledge of students. Journal for Research in Mathematics Education, 39, 372-400.

Kaput, J. (1995). Transforming algebra from an engine of inequity to an engine of mathematical power by "algebrafying" the K-12 curriculum. Paper presented at the Annual. Meeting of the National Council of Teachers Mathematics. Boston MA. 
Kaput, J. (1998). Teaching and learning a new algebra with understanding. National

Center for Improving Student Learning and Achievement in Mathematics and Science. Kaput, J. (2000). Transforming algebra from an engine of inequity to an engine of mathematical power by "algebrafying" the K-12 curriculum. National Center for Improving Student Learning and Achievement in Mathematics and Science.

Kaput, J. (2008). What is algebra? What is algebraic reasoning? En J. Kaput, D. W. Carraher y M. L. Blanton (Eds.), Algebra in the early grades (pp. 5-17). Routledge.

Kaput, J. y Blanton, M. (2001). Student achievement in algebraic thinking: A comparison of third-graders' performance on a state fourth-grade assessment. En R. Speiser, C.Maher, y C. Walter (Eds.), Proceedings of the 23rd Annual Meeting of the Qorth American Chapter of the Psychology of Mathematics Education, 1, 99-108.

Kaput, J. J., Carraher, D. W., y Blanton, M. L. (2009). Algebra in the Early Grades. Taylor \& Francis Group.

Kieran, C. (2004). Algebraic thinking in the early grades: What is it. The Mathematics Educator, 8, 139-151.

Kilpatrick, J. (2011). En J. Cai y E. Knuth (Eds.), Early algebraization. A global dialogue from multiple perspectives (pp. 125-130). Springer-Verlag.

Krippendorff, K. (1990). Metodología de análisis de contenido. Teoría y Práctica. Ediciones Paidós ibérica, S.A.

Lins, R. y Kaput, J. (2004). The early development of algebraic reasoning: The current state of the field. En K. Stacey, H. Click, M. Kendal (Eds.), The future of the teaching and learning of algebra. Proceedings of the 12th ICMI study conference (pp.47-70). Kluwer Academic Publishers.

Ministerio de Educación (2012). Bases Curriculares 2012: Educación Básica Matemática. Unidad de Curriculum y Evaluación.

Ministerio de Educación (2018). Bases Curriculares 2018: Educación Parvularia. Unidad de Curriculum y Evaluación.

Molina, M. (2009). Una propuesta de cambio curricular: integración del pensamiento algebraico en educación primaria. PNA, 3(3), 135-156.

NCTM (2003). Principios y estándares para la educación matemática. Sociedad Andaluza de Educación Matemática Thales.

NRC (2001). Adding It Up: Helping Children Learn Mathematics. The National Academies Press.

Radford, L. (2011). Embodiment, perception and symbols in the development of early algebraic thinking. En Ubuz, B. (Ed.), Proceedings of the 35th Conference of the International Group for the Psychology of Mathematics Education (vol. 4, pp. 17-24). PME. 
Rico, L., Díez, A., Castro, E. y Lupiáñez, J. L. (2011). Currículo de matemáticas para la educación obligatoria en España durante el periodo 1945-2011. Siglo XXI, 29(2), 139-172.

Rico, L. y Fernández-Cano, A. (2013). Análisis didáctico y metodología de investigación. En L. Rico, J. L. Lupiáñez y M. Molina (Eds.), Análisis didáctico en educación matemática (pp.1-22). Comares.

Singapore, Republic of. Ministry of Education (2012). Mathematics Syllabus: Primary on to six. Curriculum Planning and Development Division. Ministry of Education.

Singapore, Republic of. Ministry of Education (2013). Nurturing Early Learners: A Curriculum for Kindergartens in Singapore: Numeracy: Volume 6. Ministry of Education.

Vergnaud, G. (1988). Long terme et court terme dans l'apprentissage de l'algebre. In C. Laborde (Eds.), Actes du premier colloque franco-allemand de didactique des mathematiques et de l'informatique (pp. 189-199). La Pensée Sauvage.

Zapatera, A. (2018). Introducción del pensamiento algebraico mediante la generalización de patrones. Una secuencia de tareas para Educación Infantil y Primaria. Números. Revista de Didáctica de las matemáticas, 97, 51-67.

NATALY PINCHEIRA HAUCK

Dirección: Universidad de Girona. Facultad de Educación y Psicología.

Departamento de Didácticas Específicas 